\title{
Outcome of Abdominal Sacrocolpopexy for Post Hysterectomy Vaginal Vault Prolapse
}

\author{
BRIG. GEN. LIZA CHOWDHURY ${ }^{1}$, NURUN NAHAR KHANAM ${ }^{2}$, MAJ. JUNNU RAYEN JANNA ${ }^{3}$
}

\begin{abstract}
:
Objective (s): The aim of this study was to explore the outcome of abdominal sacrocolpopexy for the correction of post hysterectomy vaginal vault prolapse.

Materials and Methods: This prospective study was done over the period of five years from 2011 to 2015 where twenty patients of vault prolapse were subjected to abdominal sacrocolpopexy. Procedure was completed by securing the vaginal apex to the anterior longitudinal ligament of sacrum using synthetic mesh. Intra and postoperative complications and patients' satisfaction was assessed.

Results: No post-operative serious complications were reported during follow up period. The vaginal vault was well supported in all patients with no recurrent vault prolapse. One patient had mild asymptomatic rectocele. No mesh complication was found during the follow up period.

Conclusion: The abdominal sacrocolpopexy achieves excellent correction of post hysterectomy vaginal vault prolapse with minimal morbidity.
\end{abstract}

Keywords: Abdominal sacrocolpopexy, vaginal vault prolapse, anterior longitudinal ligament.

\section{Introduction:}

Where the top of the vagina gradually falls toward the vaginal opening and eventually may protrude out of the body through vaginal opening is known as vaginal vault prolapse. The vaginal vault prolapse can be encountered in patients who had abdominal or vaginal hysterectomy.

There is no precise definition of post hysterectomy vaginal vault prolapse (PHVP); however, the International Urogynecological Association (IUGA)/ International Continence Society (ICS) joint report on female pelvic floor dysfunction defines it as 'descent of the apex of the vagina that is vaginal vault or cuff scar after hysterectomy' where vaginal cuff scar corresponds to point $C$ on the Pelvic Organ Prolapse Quantification (POP-Q) grid. ${ }^{1}$

Post hysterectomy vaginal vault prolapse (PHV) has been reported to follow $11.6 \%$ of hysterectomies performed for prolapse and $1.8 \%$ for other benign diseases. ${ }^{2} \mathrm{~A}$ large study from Austria estimated the frequency of PHVP requiring surgical repair to be between $6 \%$ and $8 \%{ }^{3}$

The vaginal vault prolapse is often associated with a feeling of backache, pelvic heaviness, pressure, fullness and something coming down. There may be vaginal discomfort, dyspareunia and impaired vaginal intercourse because of something is in the way. The patient's sexual partner may also complain that the vagina is too large. If the vaginal skin is ulcerated, there may be troublesome discharge and bleeding. 4,5

A clear understanding of the supporting mechanisms for the uterus and the vagina is important in order to make the right choice of the corrective procedure and also to minimise the risk of post-hysterectomy occurrence of vault prolapse. The aim of prolapse surgery is to restore normal vaginal supports whilst maintaining vaginal capacity and coital function.

The surgical options for the correction of vault prolapse lie between the vaginal and the abdominal approach. The choice of procedure should be based on the patient's age, co-morbidity, previous surgery and the level of physical and sexual activity. ${ }^{6}$

This article is aimed to see the outcome of abdominal sacrocolpopexy (ASC) for the correction of vaginal vault prolapse encountered by hysterectomy.

\section{Materials and Methods:}

This observational study was carried out during the period of January 2011 through December 2015

1. Advisor Specialist, Obs. \& Gynae Dept, CMH, Dhaka.

2. Associate Professor, Dept. of Obs. \& Gynae, BSMMU, Dhaka.

3. Classified Specialist, Obs. \& Gynae Dept, CMH, Dhaka.

Address of correspondence: Brig Gen Liza Chowdhury, Advisor specialist, Obs \& GynaeDept, CMH, Dhaka. Mobile: 0181922050. e-mail: dr.lizachy@gmail.co 
prospectively, where twenty (20) patients who suffered from post hysterectomy vaginal vault prolapse were candidate for abdominal sacrocolpopexy (ASC) at Combined Millitary Hospital of Dhaka and Jessore and at Hi-tech Multicare Private Hospital, Dhaka. History, physical examination and personal information were recorded at the pre operation stage using questionnaire and medical chart review. This included presenting symptoms, associated problems and length of vagina at presentation.

During operation, the patient was placed in the lithotomy position. A pfannenstiel incision was made in the abdominal wall. After entering peritoneal cavity, the bowel was packed into the upper abdomen and a sufficient visual field was made.

The vaginal cuff was recognized by the aid of an assistant vaginally to elevate and manipulate the vaginal apex in the pelvis. The vaginal apex was grasped and the peritoneum overlying the vaginal apex was then incised and dissected from the anterior and posterior vaginal wall for a distance of 1 to $2 \mathrm{~cm}$.

A synthetic polypropylene mesh of $4 \times 2 \mathrm{~cm}$ (Ultrapromonocryl prolene composite) was taken and made it $Y$ shaped. Then, the vaginal tissue was tied with the mesh anteriorly and posteriorly up to $2 \mathrm{~cm}$ by giving three separate stitches. After dissecting the peritoneum overlying the sacrum the straight end of mesh was fixed to the anterior longitudinal ligament at the level of sacrum two and three. Following that peritonisation completed and buried the mesh under it. Finally, the abdominal incision was closed and as the final stage posterior colpoperineorraphy was done as and when needed.

All surgeries were done by same surgeon. Intra and postoperative complications were recorded. In postoperative period, vital signs were monitored and urinary catheter was kept continuously for 5 days. Patients were discharged at $6^{\text {th }}$ postoperative day. To track the experienced complications, the patients were instructed to come at follow up at 6 weeks, 3 months and then one year interval or whenever she feels any related problem.

Main outcome measure was relieving of distressing symptoms with restoration of normal vaginal anatomy and maintenance of bowel, bladder and sexual function.

\section{Results:}

Twenty patients were observed during the study period and there was no drop out for long 5 years. The age of studied patients was $62.80 \pm 7.35$ years ranged from 50 to 79 years with most frequent age group was 60 to 69 years. Most of them were sexually active women. The time interval of hysterectomy and vaginal vault repair was from 5 to 10 years. Majority of the patients participated in the study did not have any medical problems, only a few of them had some metabolic disorders. Among 20 patients with vaginal vault prolapse, $12(60 \%)$ patients had undergone abdominal hysterectomy and $8(40 \%)$ patients had history of vaginal hysterectomy (Table 1 ). Within preoperative symptoms in studied group, majority $(80 \%)$ had complaints of vaginal bulge and pelvic heaviness followed by dis-satisfactory sexual intercourse in $70 \%$ patients (Table 2).

Average operating time was $90.50 \pm 7.91$ minutes with average blood loss of $82.10 \pm 6.08 \mathrm{ml}$. Five patients needed posterior colpoperineorraphy due to associated rectocele. Duration of stay in hospital was 3 to 5 days.

All 20 patients got relieved from symptoms. No serious complications were reported during follow up period of one to five years except in two patients where peroperative bleeding was more than average and who needed blood transfusion. However, minor complications including urinary retention were experienced by one patient and one superficial wound infection was there. The vaginal vault was well supported in all patients with no recurrent enterocele or vault prolapse. One patient had mild asymptomatic rectocele. No mesh complication was found during the follow up period.

Table I

Characteristics of study population: $(N=20)$

\begin{tabular}{lcc}
\hline Parameters & Mean & \pm SD \\
\hline Age (Yrs) & 62.80 & \pm 7.35 \\
Weight (Kg) & 67.20 & \pm 7.22 \\
Parity & N & $\%$ \\
P4 & 8 & 40 \\
P6 & 12 & 60 \\
Causes of hysterectomy & & \\
$\quad$ Fibroid Uterus & 6 & 30 \\
$\quad$ Abnormal vaginal & 4 & 20 \\
$\quad$ bleeding & & \\
$\quad \begin{array}{l}\text { Adenomyosis } \\
\quad \text { Uterine prolapse }\end{array}$ & 2 & 10 \\
Type of hysterectomy & 8 & 40 \\
$\quad$ Total abdominal & & \\
hysterectomy & 12 & 60 \\
$\quad$ Vaginal hysterectomy & 8 & \\
Medical condition & & \\
$\quad$ Hypertension & & \\
$\quad \begin{array}{l}\text { Diabetes Mellitus } \\
\text { Bronchial Asthma }\end{array}$ & 2 & 20 \\
\hline
\end{tabular}


Table-II

Patient symptoms at presentation: $(N=20)$

\begin{tabular}{lcc}
\hline & $\mathrm{N}$ & $\%$ \\
\hline Vaginal bulging and protrusion & 16 & 80 \\
with heaviness & & \\
Backache \& Constipation & 8 & 40 \\
Recurrent urinary tract infection & 6 & 30 \\
Lower abdominal pain & 8 & 40 \\
Vaginal discharge & 2 & 10 \\
Dis-satisfactory intercourse & 14 & 70 \\
\hline
\end{tabular}

Table-III

Outcome of operation among participants: $(N=20)$

\begin{tabular}{lcc}
\hline Parameters & $\mathrm{N}$ & $\%$ \\
\hline Relieve of symptoms & 20 & 100 \\
Per-operative bleeding & 2 & 10 \\
Post-operative complications & & \\
$\quad$ Urinary retention & 1 & 5 \\
$\quad$ Superficial wound infection & 1 & 5 \\
$\quad$ Rectocele & 1 & 5 \\
\hline
\end{tabular}

\section{Discussion:}

Vaginal vault prolapse is not an uncommon complication following hysterectomy with negative impact on women's quality of life due to associated urinary, anorectal and sexual dysfunction. A clear understanding of the supporting mechanism for the uterus and vagina is important in making the right choice of corrective procedure.

This study was carried out prospectively from the year 2011 through 2015, where twenty women underwent primary repair of post-hysterectomy vaginal vault prolapse. In this study population, 8 cases of vault prolapse developed following hysterectomy for uterine prolapse and 12 cases following hysterectomy for other reasons.

There is no consensus on the role of hysterectomy as a cause of subsequent development of pelvic organ prolapse. The cumulative risk of prolapse rises from $1 \%$ to $5 \%$ after 3 and 15 years of hysterectomy respectively. Also the risk of prolapse following hysterectomy is 5.5 times in women whose initial hysterectomy was associated with genital prolapse as opposed to other reasons. Some studies have reported an incidence of up to $43 \% 6,7$

The primary aim of surgical treatment are the restoration of normal vaginal anatomy, improvement in vaginal bulge symptoms and the restoration/ maintenance of normal bladder, bowel and sexual function, ${ }^{8}$ which was aimed in our study also.

A recent qualitative study based on patient interviews showed that women are most affected by the actual physical symptoms of prolapse (bulge, pain and bowel problems) as well as by the impact that prolapse has on their sexual function. ${ }^{9}$

Current study also supports this view with most common presentation being the vaginal bulge and pelvic heaviness followed by sexual dysfunction, lower abdominal pain and constipation.

A variety of procedures exist both abdominal and vaginal for surgical treatment of PHVP in women who are deemed fit for surgery. There is no robust evidence to guide the clinician as to the best surgical technique for a particular patient. In a long-term follow up study, which compared abdominal versus vaginal route for the treatment of pelvic support defects found that reconstructive pelvic surgery for correction of significant pelvic support defects was more effective with an abdominal approach. ${ }^{10}$

Abdominal sacrocolpopexy (ASC) involves apical suspension of the vault with a permanent mesh fixed to the longitudinal ligament of the sacrum; typically, the mesh is attached to the anterior and posterior aspects of the vault with possible 'mesh extension' to correct prolapse in other compartments. ${ }^{11} \mathrm{~A}$ systematic review of observational studies reported long-term success rates of $78-100 \%$. Mesh erosion was observed in $2-11 \%$. Serious complications such as bowel injury, sacral myelitis and severe bleeding have an estimated incidence of $2 \%$ (range $0-8 \%$ ). ${ }^{12}$ To avoid mesh erosion, the biologic graft : allograft fascia lata has been used as an alternative to mesh, but resulted in unexpected failures in which no mesh could be seen during reoperation. ${ }^{13-16}$

In this study we adopted the same procedure of ASC using permanent synthetic polypropylene mesh. Results of study reported satisfaction rate of $100 \%$ in terms of relieving symptoms of vault prolapse with restoration of normal sexual activity with no bladder or bowel injury, sacral myelitis. Average per-operative bleeding was $82.10 \pm 6.08 \mathrm{ml}$ and in two cases blood transfusion was needed due to moderate bleeding. There was no mesh complication found in this study during the follow up period. 
A recent high quality RCT with 7 years' follow-up after ASC showed that pelvic organ prolapse (POP) failure rates gradually increased over the follow-up period; however, of the $10 \%$ anatomical POP failures, onehalf of the women were asymptomatic and were not retreated. Conversely, $9 \%$ were symptomatic failures, of which one-half did not meet the anatomical failure criteria. ${ }^{17}$

In our study, follow up period was expanded from one year to five year and found that

the vaginal vault was well supported in all patients with no recurrent enterocele or vault prolapse. One patient had mild asymptomatic rectocele where reoperation was not needed.

\section{Conclusion:}

A variety of procedures exist for the surgical management of vaginal vault prolapse. Successful treatment requires thorough knowledge of the anatomy, methods of diagnosis, and treatment options. The abdominal sacrocolpopexy achieves excellent correction of post hysterectomy vaginal vault prolapse with minimal morbidity.

\section{References:}

1. Haylen BT, de Ridder D, Freeman RM, Swift SE, Berghmans B, Lee J, et al.; International Urogynecological Association; International Continence Society. An International Urogynecological Association (IUGA)/ International Continence Society (ICS) joint report on the terminology for female pelvic floor dysfunction. NeurourolUrodyn2010;29:4-20.

2. Marchionni M, Bracco GL, Checcucci V, Carabaneanu A, Coccia EM, Mecacci F, et al. True incidence of vaginal vault prolapse. Thirteen years of experience. J Reprod Med 1999;44:679-84

3. Aigmueller T, Dungl A, Hinterholzer S, Geiss I, Riss P. An estimation of the frequency of surgery for posthysterectomy vault prolapse. Int Urogynecol J 2010;21:299-302.

4. Kleeman SD, Karra MM. Vaginal vault prolapse. In: Rock JA, Jones HW, editors. TeLinde's operative gynecology. 10th ed. Philadelphia: Lippincott Williams \& Wilkins; 2008. p. 911-35.

5. Zimmerman CW. Pelvic organ prolapse: basic principles. In: Rock JA, Jones HW, editors. TeLinde's operative gynecology. 10th ed. Philadelphia: Lippincott Williams \& Wilkins; 2008. p. 854.
6. Flynn BJ, Webster GD. Surgical management of the apical vaginal defect. Current Opinion in Urology. 2002;12(4):353-58

7. De Lancey JOL. Anatomic aspects of vaginal eversion after hysterectomy. American Journal of Obstetrics \& Gynecology. 1992;166(6, part 1):1717-28.

8. Post-Hysterctomy vaginal vault prolapse: Greentop guideline No.46; RCOG/BSUG joint guidelines, July 2015.

9. Srikrishna S, Robinson D, Cardozo L, Cartwright $R$. Experiences and expectations of women with urogenital prolapse: a quantitative and qualitative exploration. BJOG 2008;115:1362-8.

10. Vaginal versus abdominal reconstructive surgery for the treatment of pelvic support defects: a prospective randomized study with long term outcome evaluation. Am J Obstet Gynecol, 1996;175(6): 1418-22.

11. Marinkovic SP, Stanton SL. Triple compartment prolapse: sacrocolpopexy with anterior and posterior mesh extensions. BJOG 2003;110:323-6.

12. Nygaard IE, McCreery R, Brubaker L, Connolly A, CundiffG, Weber AM, et al.; Pelvic Floor Disorders Network. Abdominal sacrocolpopexy: a comprehensive review. Obstet Gynecol 2004;104:805-23.

13. FitzGerald MP, Edwards SR, Fenner D. Mediumterm follow up on use of freeze-dried, irradiated donor fascia for sacrocolpopexy and sling procedures International Urogynecology Journal. 2004;15(4):238-42.

14. FitzGerald MP, Mollenhauer J, Bitterman P, Brubaker L. Functional failure of fascia lata allografts. Am J Obstet Gynecol 1999;181: 1339-46.

15. FitzGerald MP, Mollenhauer J, Brubaker L. Failure of allograft suburethral slings. B J Urol 1999;84:785-5.

16. FitzGerald MP, Mollenhauer J, Brubaker L. The fate of rectus fasci suburethral slings. Am J Obstet Gynecol 2000;183:964-6.

17. Nygaard I, Brubaker L, Zyczynski HM, Cundiff G, Richter H, Gantz M, et al. Long-term outcomes following abdominal sacrocolpopexy for pelvic organ prolapse. JAMA 2013;309:2016-24. 